\title{
QUANTITATIVE CORRELATION OF CAROTID ARTERY INTIMA MEDIA THICKNESS WITH SEVERITY OF OCCLUSIVE CORONARY ARTERY DISEASE
}

\author{
Mayank Sarawag', Manju Bhaskar ${ }^{2}$
}

${ }^{1}$ Associate Professor, Department of Medicine, Jhalawar Medical College, Jhalawar, Rajasthan, India.

${ }^{2}$ Associate Professor, Department of Psychiatry, Mahatma Gandhi Medical College, Jaipur, Rajasthan, India.

\begin{abstract}
BACKGROUND
ABSTRACT

Patients with established stroke and coronary artery disease (CAD) have relatively thick carotid intima media compared to general population. There are several studies reporting the role of carotid arteries' intima media width as an independent risk factor for stroke and CAD. It shows diffuse ongoing atherosclerosis process affecting coronary and carotid as well as other arteries of the body. Carotid intima media thickness is a good and valid marker for atherosclerosis and has the potential to detect cardiovascular diseases. It reflects the collective impact of atherosclerosis in blood vessels independent of the underlying cardiovascular determinants. We wanted to assess of intima media's thickness of carotid artery and its association with CAD and stroke and correlate carotid artery intima media thickness with severity of occlusive coronary artery disease as evidenced by coronary angiography, quantitatively.
\end{abstract}

\section{METHODS}

One hundred and fifty subjects from the indoor and outdoor departments of the hospital were recruited in the present case control study. A convenient sample size of fifty was taken in each group. In the first group 50 patients of CAD, in the second group 50 patients with known CVA (Stroke) and the third group with 50 healthy controls were included. After confirming diagnosis of stroke and CAD, patients and control subjects were subjected to measurement of intima media width bilaterally (posterior wall of distal CCA). To see the extent of the disease, patients with CAD underwent coronary angiography. B-mode ultrasound scan (7.5 MHz probe) was used for measurement of intima media thickness and to see ulceration, stenosis of lumen, and plaques Doppler color scan was used. Statistical analysis was done by using SPSS software version 16. Mean, standard deviation, frequency, Chi Square and one-way ANOVA with post hoc test were used. A p value of less than 0.05 was taken as significant.

\section{RESULTS}

In present study, three study groups did not differ in age, male to female ratio, and in other socio-demographic details to avoid confounding factors. Average carotid intima media thickness in healthy controls was one millimeter; in stroke group, $1.3 \mathrm{~mm}$ and in coronary artery disease was $1.3 \mathrm{~mm}$. Carotid IMT is a determinant of atherosclerosis increased value of the width is seen in CAD and stroke affected individuals. As the Carotid IMT value goes above $1 \mathrm{~mm}$ chances of stroke and CAD would rise. When the number of blood vessels involved in CAD increases, average CIMT also increases. This concludes that more severe lesions in coronary vessels imply more severe atherosclerosis. Coronary intima media thickness is helpful in quantifying coronary atherosclerosis.

\section{CONCLUSIONS}

It is concluded that the non-invasive procedure of carotid B-mode ultra-sonography is suitable for use as a surrogate end point to measure atherosclerotic burden in the subjects because of its easy applicability. Thus, Carotid IMT is a useful, simple, cost effective and non-invasive tool to evaluate severity of atherosclerosis in patients with stroke and CAD. The pre-occlusive phase is the potential stage to retard the atherosclerosis by timely intervention as primary prevention.

HOW TO CITE THIS ARTICLE: Sarawag M, Bhaskar M. Quantitative correlation of carotid artery intima media thickness with severity of occlusive coronary artery disease. J. Evolution Med. Dent. Sci. 2019;8(15):1212-1215, DOI: 10.14260/jemds/2019/268

\section{BACKGROUND}

An increased carotid media intima thickness relates to future vascular events and Bots M.L. ${ }^{1}$ gives evidence to use the carotid media thickness measurements as an intermediate point in the observational trials.

Graner M., et $\mathrm{al}^{2}$ study concluded the association of width of intima media with the severity of CAD as assessed by quantitative coronary angiography. Assessment of the carotid

'Financial or Other Competing Interest': None.

Submission 05-02-2019, Peer Review 29-03-2019,

Acceptance 05-04-2019, Published 15-04-2019.

Corresponding Author:

Dr Manju Bhaskar

D-858, Malviya Nagar,

Jaipur, Rajasthan, India.

E-mail: manjubhaskar123@gmail.com

DOI: $10.14260 /$ jemds $/ 2019 / 268$

(c) (i) (\$) $\odot$ and coronary artery atherosclerosis in known or suspected CAD patients was done using the B-mode ultrasound and quantitative coronary angiography. Maximum and mean Carotid IMT values correlated with CAD extent, severity and atheroma burden. In conclusion, their study showed that increased carotid IMT is associated with more severe and extensive CAD. Hoskote S. S., et $\mathrm{al}^{3}$ investigated the importance of carotid intima media width as a non-invasive index of an individual's vascular health and came to the conclusion that routine screening for atherosclerosis should include Carotid intima media thickness assessment and Carotid Doppler. Hodis $\mathrm{H} \mathrm{N}$, et $\mathrm{al}^{4}$ investigated the impact of Carotid intima-media width on cardiovascular clinical events. one of the studies, concluded that for every $0.03 \mathrm{~mm}$ rise in the carotid IMT per year, there was a risk of non-fatal myocardial infarction by 2.2 and a relative risk of coronary events by 3.1. The absolute thickness and increase in the thickness of Carotid intima-media predicted the risk for a 
coronary event even more than that predicted by assessment of lipid profiles and atherosclerosis. B-mode USG score measurement of progression of intima-media thickness in the common carotid artery is found to be as useful as any other conventional risk factors to identify patients with CAD. Geroulakos G, et al ${ }^{5}$ found carotid IMT as a marker of coronary vascular disease that can be assessed noninvasively and used as marker of CAD in clinical trials. Additionally, increased IMT have significance for coronary atheroma as a screening tool. Vemmos K. N., et al $^{6}$ studied relationship of Common carotid IMT in cases with Intracerebral Hemorrhage Brain Infarction. They came to the conclusion that increased intima-media width of Common carotid (CCA-IMT), is an early marker for atherosclerosis, associated with increased risk of myocardial infarction and stroke. There wasn't any evidence of association of intima media width with cerebral bleeding.

Lorenz M.W., et $\mathrm{al}^{7}$ studied the prediction of clinical vascular events with intima-media thickness. Carotid IMT is a good predictor of future cardiovascular and cerebrovascular events. They suggested that in future IMT studies, ultrasound protocols should be aligned with published studies. Bots M.L. ${ }^{8}$ aimed to substantiate the evidence supporting the use of measurement of CIMT as a surrogate marker for cardiovascular risk and atherosclerosis.

\section{Aims and Objectives}

1. Assessment of intima media's thickness of Carotid artery and its association with CAD and stroke.

2. Quantitative correlation of the Carotid artery intima media's thickness with the severity of occlusive CAD as evidenced by coronary angiography.

\section{METHODS}

The present case control study was done in K. S. Hegde medical college and hospital, Mangalore, Karnataka under Medicine department. Institutional ethical committee approval was taken. Study was carried out from November 2008 to June 2010. Written informed consent was taken. Convenient sample size of fifty was taken in each group. Fifty cases of coronary artery disease, fifty cases of stroke and fifty healthy controls were selected for the present study using purposive sampling method. Inclusion criteria for cases includes age above 30 years, past and present diagnosis of stroke /CAD. Cases who refused to give consent were excluded from the study. Inclusion criteria for healthy controls were age above 30 years without present and past history of stroke and CAD. Blood pressure, cardiovascular status, and body mass index were noted. Laboratory assessments conducted were lipid profile and blood sugar levels. Subjects were assessed for presence of any cardiovascular risk factors (Like dyslipidemia, any history of CAD in family - particularly premature, current smoking, diabetes mellitus, and hypertension).

Diagnosis of CAD was labeled on the basis of clinical examination, ECG, cardiac enzymes, coronary angiography and any changes on ECHO. The diagnosis of stroke was done on the basis of examination, and neuro-imaging. After the confirmed diagnosis of patients, measurements of intima media thickness at the posterior wall of distal CCA were conducted. Angiography was conducted in the patients with CAD to find the extent of their disease. Healthy controls also underwent measurements of IMT. Measurement of IMT was done by using B-mode USG scan (7.5 $\mathrm{MHz}$ probe). And to see ulcerations, stenosis and plaques Colour Doppler scan was used. Carotid-artery scanning performed with high resolution Sonos 5500 (Hewlett Packard, Inc., Anaheim, CA, USA) with a duplex B-mode scanner and a linear phased array transducer of 7.5 MHz frequency were used.

Statistical analysis used SPSS software version 16.

Statistical tools used were standard deviation, mean, frequency, Chi Square and ANOVA - one way, with post hoc test. A p value of less than 0.05 was taken as significant.

\section{RESULTS}

There wasn't any difference in the three groups that were studied here with respect to age, gender, smoking, diabetes mellitus, hypertension, and family history of CVD, except that in dyslipidemia. As shown in table 1, 2, 3 and table 4, Carotid IMT is increased in risk group. There was significant increase in carotid media thickness on both sides compared to control group in risk group (CAD/Stroke). Insignificant difference in the carotid IMT in CAD groups was there, which concluded that increased intima media width is a determinant of coronary disease. This study found statistically significant increase in carotid IMT when compared in controls and CAD group. There was statistically insignificant difference in carotid IMT on either side in comparison of CAD and stroke group. (Table 4)

As in table 5, 6 a statistically significant increase in the carotid IMT on either side compared to multi-infarct stroke and other type of stroke was observed. Though it cannot be extrapolated as the sample size taken is small. The Carotid IMT of hemorrhagic stroke is more than any other stroke type but this cannot be considered significant as there were very few patients in hemorrhagic stroke group. When the average carotid IMT raises above 1-millimeter risk of atherosclerotic diseases increase. But in age group of more than 75 years this is not true as age is also very important determinant of atherosclerosis.

\begin{tabular}{|c|c|c|}
\hline No. of Vessels Involved & & CAD Group \\
\hline \multirow[t]{2}{*}{0} & Count & 2 \\
\hline & $\%$ & $4.0 \%$ \\
\hline \multirow[t]{2}{*}{1} & Count & 24 \\
\hline & $\%$ & $48.0 \%$ \\
\hline \multirow[t]{2}{*}{2} & Count & 15 \\
\hline & $\%$ & $30.0 \%$ \\
\hline \multirow[t]{2}{*}{3} & Count & 9 \\
\hline & $\%$ & $18.0 \%$ \\
\hline \multirow[t]{2}{*}{ Total } & Count & 50 \\
\hline & $\%$ & $100.0 \%$ \\
\hline \multicolumn{3}{|c|}{ Table 1. Vessel Involvement in CAD Group } \\
\hline
\end{tabular}




\begin{tabular}{|c|c|c|}
\hline Type of Stroke & & \\
\hline \multirow[t]{2}{*}{ Bleed } & Count & 4 \\
\hline & $\%$ & $8.0 \%$ \\
\hline \multirow[t]{2}{*}{ Multi Infarct } & Count & 1 \\
\hline & $\%$ & $2.0 \%$ \\
\hline \multirow[t]{2}{*}{ Post. Circulation Stroke (Infarct) } & Count & 2 \\
\hline & $\%$ & $4.0 \%$ \\
\hline \multirow[t]{2}{*}{ Ant. Circulation Stroke (Infarct) } & Count & 43 \\
\hline & $\%$ & $86.0 \%$ \\
\hline \multirow[t]{2}{*}{ Total } & Count & 50 \\
\hline & $\%$ & $100.0 \%$ \\
\hline \multicolumn{3}{|c|}{ Table 2. Type of Stroke } \\
\hline
\end{tabular}

\begin{tabular}{|c|c|c|c|}
\hline Stroke Group & Pattern of Weakness & Frequency & Percent \\
\hline \multirow{4}{*}{} & None (TIA) & 3 & 6.0 \\
\cline { 2 - 4 } & Lt. Hemiparesis (Bleed) & 2 & 4.0 \\
\cline { 2 - 4 } & Lt. Hemiparesis (Infarct) & 19 & 38.0 \\
\cline { 2 - 4 } & Multi Infarct stroke & 1 & 2.0 \\
\cline { 2 - 4 } & Posterior Circulation Stroke (Infarct) & 8.0 \\
\cline { 2 - 4 } & Rt. Hemiplegia (Bleed) & 19 & 4.0 \\
\cline { 2 - 4 } & Rt. Hemiplegia (Infarct) & $\mathbf{5 0}$ & 38.0 \\
\cline { 2 - 4 } & Table 3. Stroke Pattern in Stroke Group & $\mathbf{1 0 0 . 0}$ \\
\hline \multicolumn{2}{|c|}{} \\
\cline { 2 - 4 }
\end{tabular}

\begin{tabular}{|c|c|c|c|c|c|c|}
\hline \multicolumn{2}{|c|}{} & Number of Participants & Mean CIMT (mm) & Standard Deviation & F & p \\
\hline Right Side CIMT & Control Group & 50 & 1.0700 & .20329 & & \\
\hline & Stroke Group & 50 & 1.3000 & .22857 & & \\
\hline & CAD Group & 50 & 1.3020 & .17437 & 21.522 & $<0.001$ \\
\hline Left Side CIMT & Control Group & 50 & 1.0540 & .20919 & & \\
\hline & Stroke Group & 50 & 1.2900 & .25173 & & \\
\hline & CAD Group & 50 & 1.2800 & .20304 & 18.010 & $<0.001$ \\
\hline \multicolumn{7}{|l}{} \\
\hline
\end{tabular}

\begin{tabular}{|c|c|c|c|c|}
\hline Dependent Variable & (I) Group & (J) Group & Mean Difference (I-J) & \\
\hline Rt. CIMT & Control Group & Stroke Group & -0.2300 & $<0.001 \mathrm{vhs}$ \\
& & CAD Group & -0.2320 & $<0.001 \mathrm{vhs}$ \\
& & CAD Group & -0.0020 & $.999 \mathrm{~ns}$ \\
\hline & Stroke Group & Stroke Group & -0.2360 & $<0.001 \mathrm{vhs}$ \\
\hline Lt. CIMT & Control Group & CAD Group & -0.2260 & $<0.001 \mathrm{vhs}$ \\
\hline & & CAD Group & 0.0100 & $.973 \mathrm{~ns}$ \\
\hline
\end{tabular}

\begin{tabular}{|c|c|c|c|}
\hline CAD Group & Vessel Involvement & Frequency & Percent \\
\hline \multirow{4}{*}{} & DVD (LAD, LCX) & 6 & 12.0 \\
\cline { 2 - 4 } & DVD (RCA, LAD) & 4 & 10.0 \\
\cline { 2 - 4 } & DVD (RCA, LCX) & 5 & 4.0 \\
\cline { 2 - 4 } & Non-Critical CAD & 2 & 16.0 \\
\cline { 2 - 4 } & SVD (LCX) & 8 & 14.0 \\
\cline { 2 - 4 } & SVD (RCA) & 7 & 18.0 \\
\cline { 2 - 4 } & SVD (LAD) & 9 & 18.0 \\
\cline { 2 - 4 } & TVD & $\mathbf{5 0}$ & $\mathbf{1 0 0 . 0}$ \\
\cline { 2 - 4 } & \multicolumn{2}{|c|}{ Total } & \\
\hline
\end{tabular}

\begin{tabular}{|c|c|c|c|c|c|c|c|}
\hline Group & & $\begin{array}{c}\text { Number of } \\
\text { Participants }\end{array}$ & $\begin{array}{c}\text { Mean carotid } \\
\text { IMT (mm) }\end{array}$ & $\begin{array}{c}\text { Std. } \\
\text { Deviation }\end{array}$ & $\begin{array}{c}\text { Minimum } \\
\text { CIMT }\end{array}$ & $\begin{array}{c}\text { Maximum } \\
\text { CIMT }\end{array}$ \\
\hline Stroke & Rt. CIMT & Bleed & 4 & 1.32 & 0.41932 & 0.90 & 1.90 \\
\hline & & Multi Infarct & 1 & 1.60 & & 1.60 & 1.60 \\
\hline & & Post. Circulation Infarct & 2 & 1.30 & 0.14142 & 1.20 & 1.40 \\
\hline & & Ant. Circulation Infarct & 43 & 1.29 & 0.21360 & 0.90 & 1.80 \\
\hline & & Total & $\mathbf{5 0}$ & $\mathbf{1 . 3 0}$ & $\mathbf{0 . 2 2 8 5 7}$ & $\mathbf{0 . 9 0}$ & $\mathbf{1 1 . 9 0}$ \\
\hline & Lt. CIMT & Bleed & 1 & 1.35 & 0.28868 & 1.00 & 1.70 \\
\hline & & Multi Infarct & 1.40 & & 1.40 & 1.40 \\
\hline & & Post. Circulation Infarct & 2 & 1.30 & 0.14142 & 1.20 & 1.40 \\
\hline & Ant. Circulation Infarct & 43 & 1.28 & 0.25843 & 0.80 & 1.90 \\
\hline & \multicolumn{2}{|r|}{ Total } & $\mathbf{5 0}$ & $\mathbf{1 . 2 9}$ & $\mathbf{0 . 2 5 1 7 3}$ & $\mathbf{0 . 8 0}$ & $\mathbf{1 . 9 0}$ \\
\hline
\end{tabular}




\section{DISCUSSION}

In the present study baseline characteristics of the three study groups were matched. This study concluded that carotid IMT is a determinant of atherosclerosis as its value is high in CAD and stroke compared to matched healthy controls and increased value of carotid IMT is strongly associated with stroke and CAD. As risk factors for atherosclerosis, like age, dyslipidemia, hypertension, diabetes mellitus, and family history of CVD increases the value of carotid IMT increases. In our study average carotid IMT came out to be $1.0 \mathrm{~mm}$ in healthy controls, $1.3 \mathrm{~mm}$ in stroke group and $1.3 \mathrm{~mm}$ in CAD group too. When the average carotid IMT rises more than one $\mathrm{mm}$ risk of atherosclerotic diseases like CAD and stroke rises. In CAD group when the number of vessels involved increases the average carotid IMT increases. This concludes that more severe the lesion in the coronary bed, severe is the atherosclerosis. Thus, it can be said that carotid IMT quantifies coronary artery disease.

Gupta $H$. et al $^{9}$ conducted a study to determine the association between intima-media width and CAD risk factors concluding that intima media thickness was significantly more in patients with coronary artery disease. On multivariate logistic regression analysis, carotid IMT was found to be an independent determinant of CAD. They also got significant association between cardiovascular disease risk factors and carotid IMT in both controls and CV diseased group. Similar results were found in our study, but we had three groups.

Another study from India ${ }^{10}$ found statistically significant high mean carotid IMT values in patients with diabetes as compared to non-diabetic controls. Diabetes Mellitus is found to be a very strong risk factor for atherosclerosis and we also got a strong causal association of diabetes mellitus with stroke in our study. But the strong association of diabetes with coronary artery disease among the CAD group was not established possibly due to small sample size.

Atherosclerosis Risk in Communities study, 7 found that carotid IMT was more in patients with cardiovascular risk factors compared to controls. This population-based study followed a large cohort for at least ten years and controls without risk factors for atherosclerosis were compared with people with risk factors for atherosclerosis. In present study we also compared healthy controls with risk factors for atherosclerosis with CAD and stroke groups who have both modifiable and Non-modifiable risk factors. Present study also found that when number of risk factors for atherosclerosis increases carotid media thickness increases. Previous studies also shown that carotid IMT can indicate the presence of coronary artery disease. Present study reports statistically significant association between carotid intima media thickness and the presence and severity of coronary artery disease in the Indian setting. But more data are needed to establish CIMT as a noninvasive screening tool for the detection of coronary artery disease in symptomatic or asymptomatic groups. Carotid IMT role in predicting the risk of future cardiovascular events in western countries has already done to established by several large-scale randomized studies. But carotid intima media thickness role for prediction of future cardiovascular disease has not been done on large randomized prospective studies in India. Present study was carried on small sample size with casecontrol study design, so it is difficult to generalize the data in general population. Finally, it is concluded that the noninvasive nature and easy applicability of carotid B-mode ultrasound makes it suitable for screening of atherosclerosis. Patients should undergo measurement of intima media thickness in patients with multiple cardiovascular risk factors for after cardiologist's recommendation.

\section{CONCLUSIONS}

Noninvasive nature and easy applicability of carotid B-mode ultrasound makes it suitable for screening of atherosclerosis. Patients with multiple cardiovascular risk factors should undergo measurement of intima media thickness after cardiologist's recommendation.

\section{REFERENCES}

[1] Bots ML, Hoes AW, Koudstaal PJ, et al. Common carotid intima media thickness and risk of stroke and myocardial infarction - the Rotterdam study. Circulation 1997;96(5):1432-7.

[2] Graner M, Varpula M, Kahri J, et al. Association of carotid intima-media thickness with angiographic severity and extent of coronary artery disease. American Journal of Cardiology 2006;97(5):624-9.

[3] Hoskote SS, Joshi SR. Carotid intimomedial thickness a non-invasive index of vascular health. J Assoc Physicians India 2008;56:577-8.

[4] Hodis HN, Mack WJ, LaBree L, et al. The role of carotid arterial intima-media thickness in predicting clinical coronary events. Ann Intern Med 1998;128(4):262-9.

[5] Geroulakos G, O'Gorman DJ, Kalodiki E, et al. The carotid intima-media thickness as a marker of the presence of severe symptomatic coronary artery disease. Eur Heart J 1994;15(6):781-5.

[6] Vemmos KN, Tsivgoulis G, Spengos K, et al. Common carotid artery intima-media thickness in patients with brain infarction and intracerebral hemorrhage. Cerebrovasc Dis 2004;17(4):280-6.

[7] Lorenz MW, Markus HS, Bots ML, et al. Prediction of clinical cardiovascular events with carotid intimamedia thickness: a systematic review and metaanalysis. Circulation 2007;115(4):459-67.

[8] Bots ML. Carotid intima-media thickness as a surrogate marker for cardiovascular disease in intervention studies. Curr Med Res Opin 2006;22(11):2181-90.

[9] Gupta H, Bhargava K, Bansal M, et al. Carotid intima media thickness and coronary artery disease: an Indian perspective. Asian Cardiovascular Thoracic Annuals 2003;11(3):217-21.

[10] Mohan V, Ravikumar R, Shanthirani S, et al. Intimal medial thickness of the carotid artery in South Indian diabetic \& non-diabetic subjects: the Chennai Urban Population Study (CUPS). Diabetologia 2000;43(4):494-9. 